\title{
Current Trends in Design and Development of Nanopharmaceutical Dosage Forms
}

\author{
Panda BP1*, Patnaik $\mathrm{S}^{2}$ and Maharana $\mathrm{RL}^{3}$ \\ ${ }^{1}$ Department of Pharmaceutical Technology, School of Pharmacy, Taylors \\ University, Lakeside Campus, Selangor, Malaysia \\ 2University College of Pharmaceutical Sciences, Kakatiya University, Warangal, \\ Telangana, India
}

${ }^{3}$ Department of Pharmaceutics, Roland Institute of Pharmaceutical Sciences, Berhampur, India

*Corresponding author: Bibhu Panda Prasad, School of Pharmacy, Taylor's University, Lakeside Campus, No.1, Jalan Taylor's, Jalan Taylor's, 47500 Subang Jaya, Selangor Darul Ehsan, Malaysia, Tel: +60187756120; E-mail: bibhuprasad.panda@taylors.edu.my

\section{Abstract}

Nanopharmaceuticals or Nanopharmaceutical dosage forms pertaining to design of pharmaceuticals using nanotechnology based science are intended to develop various pharmaceutical dosage forms and carrier systems in the range of 10 to $1,000 \mathrm{~nm}$. Nanopharmaceuticals offer novel reformulation opportunities in development of nanoscale pharmaceutical dosage forms, for delivering of many common therapeutic agents which produce challenges in the formulation of dosage form because of its low solubility, poor bioavailability, lack of specific site targeting or toxicity issues. Nanopharmaceutical dosage forms examples are solid lipid nanoparticles, polymeric nanoparticles, pegylated nanostructures, nanocrystals, dendrimers, nanotubes, nanofibers, nanomicelles, nanoshells, magnetic nanopharmaceuticals, metallic nanoparticles, nanosized powders, nanoemulsions and nanosuspensions. These nanopharmaceutical dosage forms are able to accommodate the active therapeutic moiety and bio-molecules such as enzymes, vaccines, recombinant proteins, peptides, and nucleic acid for their delivery. These dosage systems have ability to improve specific targeting either passively or actively, to a particular tissue or organ of the body where they are needed. The prime objective of this review article is to provide concise overview on nanopharmaceutical dosage forms and their potential applications as carrier systems in the delivery of therapeutic agent.

Keywords: Nanopharmaceutical dosage forms; Nanopharmaceutical carrier systems; Delivery and targeting of therapeutic agent

\section{Introduction}

Development of various pharmaceutical dosage forms and devices in the range of 10 to $1,000 \mathrm{~nm}$ using the concept of nanotechnology is referred as nanopharmaceuticals or nanopharmaceutical dosage forms [1]. They have emerged as one of the most promising and challenging field for research in the last few decades [2]. Many pharmaceutical and biological formulations of nanopharmaceutical products got 
approval from the Food and Drug Administration (FDA) in US. More than 1000 nanopharmaceutical based patents are issued by the U.S. Patent and Trademark Office (U.S. PTO) during the last 10 years [3]. Nanopharmaceuticals can be categorized into nanoscale either because of size of the drug particle or due to drug delivery system of therapeutic agent itself. The overall goal of nanopharmaceutical is to diagnose and treat the diseases accurately and effectively as early as possible with optimal therapeutic outcomes. Nanopharmaceuticals have huge potential in addressing the drugs which fail in formulation because of factors such as low water solubility, toxicity issues, poor bioavailability, or lack of target specificity. Nanopharmaceuticals have better pharmacokinetic profile and enhanced bioavailability of therapeutic agent to a targeting, specific to organ or tissue, which results in improved efficacy and reduce the drug toxicity by specific targeting [4]. Primarily nanopharmaceutical formulations are colloidal in nature of range 10 to $1,000 \mathrm{~nm}$ in size and widely used in drug delivery system. In comparison with macro molecular carrier system, nanoformulations offer many advantages because of its shape, composition, surface area and quantum effects of the formulation. As the particle's size decreases, particles become more reactive and more water soluble.

\section{Types of Nanopharmaceuticals}

In general there are two types of nanopharmaceuticals, (i) Therapeutic agent itself acts as own nanocarrier system and (ii)Therapeutic agent directly coupled (functionalized, entrapped, or coated) with nanoparticle carrier or nanoengineered drug.

As there is no universal nomenclature system for the classification of nanopharmaceuticals, different nanoscale structures of different shapes are often classified as nanopharmaceuticals. In fact, some of the common shapes of nanopharmaceuticals include spheres (hollow or solid), tubules, particles (solid or porous), and tree-like branched macromolecules. Some of the common shapes of nanopharmaceutical dosage forms are shown in Figure 1 and few selected nanopharmaceutical dosage forms available in the market that have been approved by the FDA are provided in Table 1. Approaches such as self-assembly, vapour or electrostatic deposition, aggregation, nanomanipulation, imprinting, etc. can be employed for synthesis of nanopharmaceuticals. Factors which influence the protocol of nanopharmaceutical formulation depend upon the specific therapeutic use of the drug and the desired route for delivery of the dosage form. The functional complexity of nanopharmaceuticals depends upon [5].

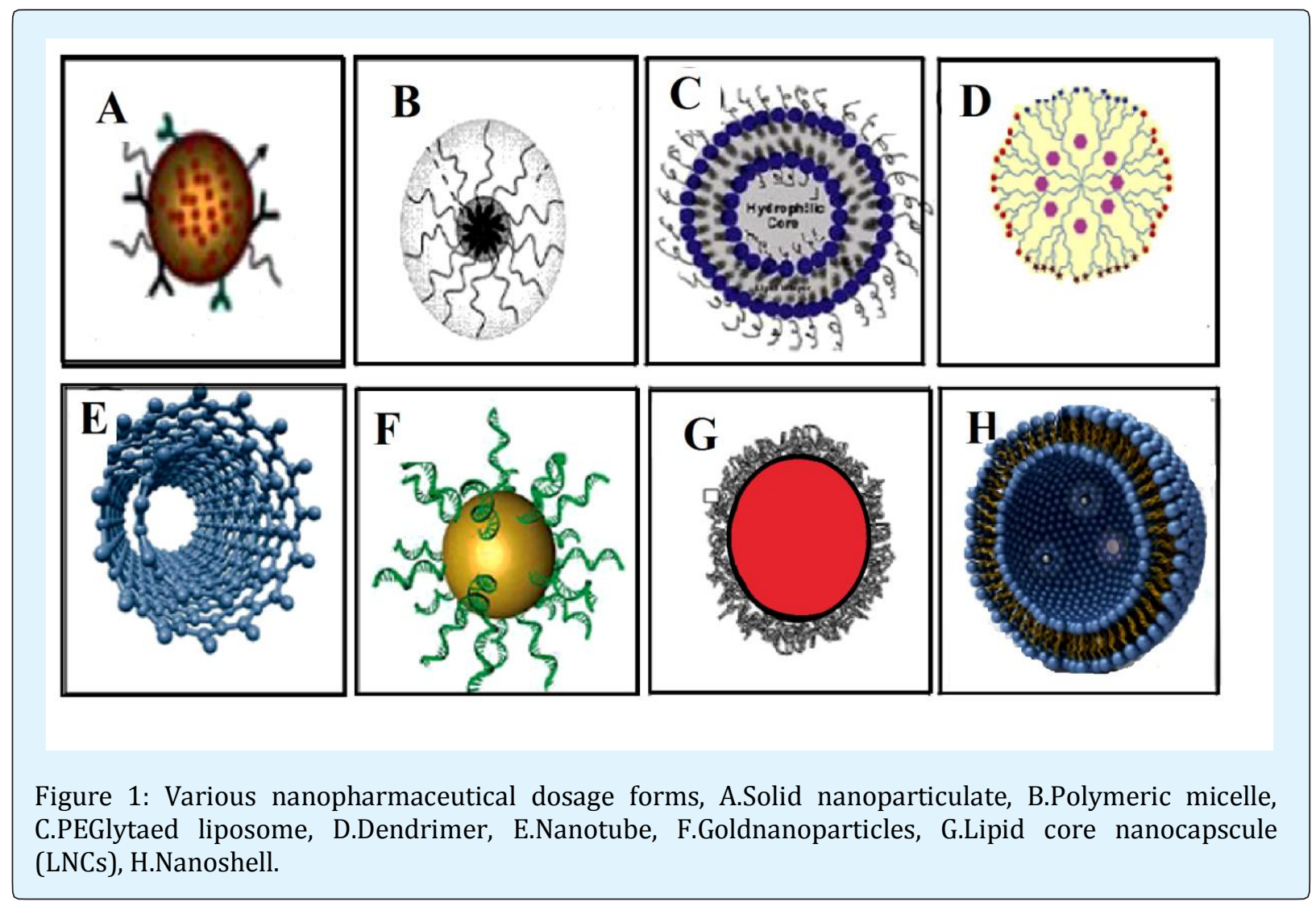




\begin{tabular}{|c|c|c|c|c|}
\hline Drug & $\begin{array}{c}\text { Formulation and } \\
\text { route of } \\
\text { administration }\end{array}$ & Brand Name & Company & Therapeutic Indications \\
\hline Amphotericin B & $\begin{array}{l}\text { Lipocomplex } \\
\text { (iv infusion) }\end{array}$ & Amphocil & $\begin{array}{c}\text { Sequus } \\
\text { Pharmaceuticals }\end{array}$ & Serious fungal infections \\
\hline Amphotericin B & $\begin{array}{l}\text { Lipocomplex } \\
\text { (iv infusion) }\end{array}$ & Ambisome & $\begin{array}{c}\text { NeXstar } \\
\text { Pharmaceuticals } \\
\end{array}$ & Serious fungal infections \\
\hline Amphotericin B & $\begin{array}{l}\text { Lipocomplex } \\
\text { (iv infusion) }\end{array}$ & Abelcet & $\begin{array}{c}\text { The Liposome } \\
\text { Company } \\
\text { (Princeton,New Jersey) }\end{array}$ & Serious fungal infections \\
\hline Paclitaxel & $\begin{array}{l}\text { Albumin bound } \\
\text { nanoparticles } \\
\text { (iv injection) } \\
\end{array}$ & Abraxane & $\begin{array}{l}\text { American Biosciences } \\
\text { (Blauvelt,New York) }\end{array}$ & Metastatic breast cancer \\
\hline Sirolimus & $\begin{array}{c}\text { Nanocrystal } \\
\text { particles(Oral) }\end{array}$ & Rapamune & $\begin{array}{c}\text { WyelthElan } \\
\text { (Madison,New Jersey) }\end{array}$ & $\begin{array}{l}\text { Immunosuppressant in } \\
\text { kidney transplant patients }\end{array}$ \\
\hline $\begin{array}{l}\text { Aprepitant, } \\
\text { MK869 }\end{array}$ & $\begin{array}{c}\text { Nanocrystal } \\
\text { particles(Oral) }\end{array}$ & Emend & $\begin{array}{c}\text { Merck/Elan } \\
\text { (Whitehouse } \\
\text { Station,New Jersey) } \\
\end{array}$ & $\begin{array}{l}\text { For chemotherapy patient to } \\
\text { delayed nausea and vomiting }\end{array}$ \\
\hline Doxorubicin & $\begin{array}{c}\text { Liposome } \\
\text { (iv injection) }\end{array}$ & Doxil & $\begin{array}{c}\text { Sequus } \\
\text { Pharmaceuticals } \\
\end{array}$ & Kaposi sarcoma in AIDS \\
\hline $\begin{array}{l}\text { Daunorubicin } \\
\text { citrate }\end{array}$ & $\begin{array}{c}\text { Liposome } \\
\text { (intravenous) }\end{array}$ & DaunoXome & $\begin{array}{c}\text { NeXstar } \\
\text { Pharmaceuticals }\end{array}$ & Kaposi sarcoma in AIDS \\
\hline
\end{tabular}

Table 1.Examples of Few Marketed Nanopharmaceutical Dosage Forms.

- $\quad$ The nature of polymeric nanomaterials used. E.g. liposomes, carbon nanotubes, dendrimers,

- Colloidal gold, nanocrystals, fullerenes

- The nature of drugs that are packaged in nanomaterials. E.g. proteins, nucleic acids, smallmolecule

- drugs,

- The nature of targeting moieties that can be surface-functionalized with nano carrier. E.g.

- Antibodies, ligands

- The route of drug delivery. E.g. oral, topical, intravenous, etc.

- The shape/geometry of nanopharmaceuticals

- The chemical composition of nanopharmaceuticals

- The nano-scale dimensions (large surface-area-tovolume ratio) of nanopharmaceuticals

- The surface charge and release properties of nanopharmaceuticals

\section{Nanocarriers for Targeting}

Nanocarriers based targeted dosage forms have been designed to reduce the clearance, to increase therapeutic index and lower the dose required for therapeutic efficacy. Typically targeted nanopharmaceuticals are accumulating non-uniformly within the body and their ultimate location of accumulation and fate of delivery are decided by their size, distribution, surface charge and surface properties. In fact, these properties can be modulated to provide long or short circulation times. Targeting to specific tissue sites such as hepatocytes versus kupffer cells in the liver, can be achieved by linking specific ligands or molecules like antibodies, glycoproteins, etc. to the polymeric carrier or altering the surface characteristics of the polymeric carrier so that it evades the reticuloendothelial (RES) system [6]. Biological prerequisites for nanopharmaceutical for effective and site specific targeting are,

- Non-toxic, biodegradable and traceable;

- Ability evade from macrophage attack and the immune response;

- Effective pharmacokinetic properties with electivity for effective targeting.

\section{Nanopharmaceutical Dosage Forms}

Solid Lipid Nanoparticles: Solid lipid nanoparticles (SLN) were evolved in the year 1990, as an alternative colloidal carrier system for emulsions, liposomes and polymeric nanoparticles in controlled drug delivery of therapeutic agents. Primarily, SLN are composed of physiological lipids and surfactants that have an accepted GRAS (Generally Recognized as Safe) grade, where the drug is normally incorporated in the submicron size range (below $1 \mu \mathrm{m}$ ). The potential advantages of solid lipid nanoparticles over polymeric nanoparticles are recognized on the basis of lower cytotoxicity, higher drug loading capacity, and best production scalability. In large scales, SLN can be produced by using high-pressure homogenization without using organic solvents. It is reported to have been used as pharmaceutical dosage form in various routes of administration such as oral, parenteral, pulmonary and dermal [7]. 
Solid lipid nanopharmaceutical is emerging with wide potential applications in the areas of drug delivery, clinical medicine and pharmaceutical research. Due to their unique size-dependent properties, lipid nanoparticles offer tremendous possibilities in the development of new therapeutics and hold great promise for attaining the bioavailability enhancement along with site specific and controlled drug delivery. Additionally, SLN help to prevent the degradation of drug molecules sensitive to outer environment such as water, light and also controlled release characteristics were achieved by incorporation of poorly water soluble drugs by formulating into solid lipid matrix. The innovative nanoparticles of solid lipid matrices coated with poly (alkylcyanoacrylate) surfactant are specifically designed for brain targeting. SLN with cationic lipids have also been considered as new transfection agents. For example, SLN prepared with a cationic lipid (DOTAP) had the same transfection efficiency as the liposomes from the same cationic lipid, but with SLN the range of strong non-viral transfection agents that can be produced in large scale is widened [8]. Solid lipid nanoparticles (SLN) loaded with doxorubicin were prepared by solvent emulsificationdiffusion method. Glyceryl caprate was used as lipid core, and curdlan as the shell material. Dimethyl sulfoxide (DMSO) was used to dissolve both lipid and drug. Polyethylene glycol 660 hydroxystearate was employed as surfactant. Major formulation parameters were optimized to obtain high quality nanoparticles. Cytotoxicity results showed that lyophilized SLN were equally effective after 1 year of storage at $4^{\circ} \mathrm{C}$. These observations suggest that present system offers an exciting mode of delivery to the lipophilic anticancer drugs for tumour targeting [9].

Polymeric Nanoparticles: The term nanoparticle is a collective name for both nanospheres and nanocapsules. Nanospheres are considered as a matrix system in which the drug is uniformly dispersed and nanocapsules are described as a polymeric membrane which surrounds the drug in the matrix core. Polymeric biodegradable nanoparticles have better efficiency and effectiveness over traditional methods of polymeric biodegradable matrix system of drug delivery. There are many approaches for production of nanoparticles, but in broader way it can be classified into two main categories according to whether the formulation requires a polymerization reaction or is achieved directly from a macromolecule or preformed polymer. List of biodegradable polymers used in polymeric nanoparticles drug delivery are chitosan, gelatin, sodium alginate, polycyanoacrylate or poly (D, Llactide) and poly (lactide-co-glycolide) (PLGA). Drug encapsulation, absorption, biodistribution pattern, elimination and drug release are affected by various factors, including polymer composition, hydrophobicity, surface charge, biodegradation profile of the nanoparticles, adjuvant substances and associated drugs. Along with controlled release property nanoparticles also improve the stability of drugs and biologicals. These particles may have specificity, allowing them to deliver a higher concentration of pharmaceutical agent to a desired location due to the possible change in surface charge or other properties (e.g., nasal and brain location). Polymeric nanoparticles are ideal candidates for cancer therapy, delivery of vaccines, contraceptives and delivery of targeted antibiotics. Moreover, polymeric nanoparticles can be easily incorporated into other systems related to drug delivery, such as tissue engineering and drug delivery for species other than humans.

A research study reveals the influence of polymeric nanoparticles with distinct physicochemical properties on the adsorption and dynamic surface tension lowering properties of pulmonary surfactant. Poly (styrene) and poly (D, L-lactide-co-glycolide) nanoparticles suggest a dose-dependent influence on biophysics of pulmonary surfactant, positively-charged nanoparticles made from poly(butyl methacrylate- $c 0$ (2-dimethylaminoethyl) methacrylate-co-methyl methacrylate) showed no effect. This behavior is attributed to the differences in zeta potential and surface hydrophobicity, which in turn involves an altered adsorption pattern of the positively charged surfactant proteins to the nanoparticles. This study establishes that polymeric nanoparticles do not substantially affect the biophysical properties of pulmonary surfactant and may be a viable drug-delivery vehicle for the inhalative treatment of respiratory diseases [10]. Another study indicated that the newly conceived amino acid based biocompatible polymeric nanoparticles might have potential application as injectable drug delivery systems, which can enhance the therapeutic index of poorly water-soluble clinically challenging drugs [11]. The adsorption or grafting of polyethylene glycol (PEG) to the surface of nanoparticles or addition of PEG and PEG-containing copolymers to the surface of nanoparticles results in an increase in the blood circulation half-life of the particles by several orders of magnitude. This method creates a hydrophilic protective layer around the nanoparticles that is able to repel the absorption of opsonin proteins via steric repulsion forces, thereby blocking and delaying the first step in the opsonization process [12].

Peglyated Nanostructures: Polyethylene glycol (PEG) or polyethylene oxide is a water-soluble material widely employed in pharmaceutical applications, as its terminal hydroxyl groups can be easily converted into reactive functional groups by a number of routine 
reactions of organic chemistry. PEGlyation has been designated as a technique of attaching PEG to any drug, peptide, polymer or other compounds and its biological applications have been well established. PEGlyation enhance the pharmacokinetics of protein and peptide drugs that could be degraded by proteolytic enzymes or have a short circulating half life. With PEGlyation, proteins and peptide drugs are shielded from proteolytic enzymes, resulting in longer circulating times, better acceptability by body tissues and improved ability to deliver drugs to the intended tissues. For example, PEGlyated nanostructure liposomes of doxorubucin has shown improved efficacy in breast cancer treatment. The next generation of liposomes for delivery systems will include molecular targeting, as in the case of immunoliposomes that PEGlyated nanostructured lipid carriers (PEG-NLCs), to improve the delivery of anti-tumour agents to lung tumours. PEG-40 NLCs modified with PEG-40 stearate (molecular weight $2000 \mathrm{Da}$ ), PEG-100 NLCs modified with PEG-100 stearate (molecular weight $5000 \mathrm{Da}$ ) and NLCs without PEG modification were prepared by meltemulsification and homogenization, and loaded with 10hydroxycamptothecin (HCPT). It was also investigated in terms of physiological characteristics, biodistribution, cellular uptake, and anti-tumour effect in-vivo. Encapsulation in PEG-NLCs protected the active lactone form of HCPT compared with HCPT solution after incubation with plasma. In biodistribution studies, PEG-NLCs, especially PEG-40 NLCs, had longer circulation time and decreased uptake by the reticuloendothelial system (RES) compared with unmodified NLCs. PEG-NLCs accumulated in the lungs after i.v. injection in mice. PEG-NLCs showed enhanced cellular uptake by human lung adenocarcinoma epithelial A549 cells. In-vivo experiments indicated that PEG-NLCs loaded with HCPT have superior efficacy against A549 lung cancer compared with HCPT solution and NLCs. These results suggest that PEG-NLCs are a promising delivery system for HCPT in the treatment of lung cancer [13].

Nanocrystals: The production of nanocrystals and nanosuspensions is called nanonization. There are several techniques to obtain this kind of nanomaterials, such as high pressure homogenization, wet milling and by nanocrystallization from supersaturated solution state or spray drying. Nanocrystals increase surface area and drug solubility, thus enhancing oral bioavailability and enabling administration by injection or infusion as intravenous aqueous solution of drugs that are poorly soluble in water. Nanocrystals are taken up by the mononuclear phagocytic system to allow regional specific delivery. It is known from the literature that these nanoparticles act very quickly when pathogens persist intracellularly, e.g., targetingantimycobacterial, fungal or leishmanicidal active macrophages [14].

The nanocrystals are administered through various routes such as oral, dermal, mucosal, ocular, pulmonary and intravenous. In addition, the potential of the nanocrystal technology for delivery of poorly soluble, non-pharmaceutical application is highlighted, i.e. in cosmetics or nutraceuticals [15].To reduce toxicity and enhancing therapeutic efficacy of paclitaxel, nanocrystal is compared with other marketed formulations and has been found to be more efficacious [16]. It is also reported that nanocrystals have the ability to improve the dissolution rate among CoQ10 nanocrystals and lipid nanoparticles [17].

Dendrimers: Dendrimers were discovered in the early 1980, and its nano formulations are at the beginning era of its commercialization. Dendrimers have a central core, internal branches and symmetrical distribution of terminal groups in three dimensions. Mono-dispersed dendrimers provide a controlled, well defined nanoscale sphere carrying multiple attachment sites and a hydrophobic interior for binding and release of hydrophobic chemicals. A dendrimer molecule has a great potential to link treatment with detection and diagnostics. The branching structure provides them enormous surface area to which therapeutic active agents or other bioactive molecules like peptides can be attached. A single dendrimer can carry a molecule that recognises tumour cells, therapeutic active agents to kill those cancer cells and a molecule that recognizes the cell death signal. The biocompatible biotin-dendrimer conjugate might be a promising nano-platform for cancer therapy and cancer diagnosis [18]. Dendrimer based multivalent methotrexate are used for cancer cell targeting [19].

Nanotubes: Carbon based materials may be advantageous in biotechnological applications for the large variety of properties and shapes that they offer. These are self-assembled lipid microtubes, fullerenes and the various types of nanotubes (carbon nanotubes, cyclic peptide nanotubes and template synthesized nanotubes). The possible chemistry and biochemistry that can be applied using the template method are given due importance. However, the issue of production cost and mass production of nanotubes must be addressed. The preparation of solid nanowires or hollow nanotubes (cylindrical nanostructures) with monodisperse diameters and lengths depends on the membrane and synthetic method used. The method is quite general to prepare nanowires and nanotubes composed of many types of material, including metals, polymers, semiconductors and carbon. Carbon nanotubes have recently emerged as a new option for 
possible use in methodologies of cancer treatment, bioengineering, gene therapy and also as a functional excipient for pharmaceutical dosage forms [20,21].

Nanofibers: Electrospinning is a process that was originally developed in the early 1930s, but did not receive much attention until recent decades. Most likely, the increased interest is due to the refocusing of more active research on nanotechnology. A typical electrospinning process involves dissolving the drug of interest and a polymer in an appropriate solvent. The solution is then placed in a syringe, and a high voltage is applied. A small amount of the polymer solution is drawn out of the syringe, forming a taylor cone. Increasing the applied voltage further results in the initiation of a charged fluid jet, which follows a chaotic trajectory of stretching and bending until it reaches the grounded target. A stable jet is formed when the charge is increased above a critical voltage, and there is a balance between the surface tension of the fluid and the repulsive nature of the charge distribution on the surface of the fluid. This process typically results in the drawing of a virtually endless fiber with a nanometersized to micrometer-sized diameter. The final product is a three-dimensional nonwoven mat of entangled nanofibers with a high surface area to volume ratio. Electrospun nanofibers have a very large surface area to volume ratio, as large as 1000 times that of a microfiber. This physical property has generated a significant amount of interest in the biomedical and pharmaceutical industries particularly for drug delivery of poorly soluble drug substances. Further utility may be found with electrospinning by embedding it at the end of the chemical synthesis of the drug substance, which would streamline the transfer of material from chemical development to pharmaceutical development. The last step in the chemical synthesis of a drug is usually a purification step to create a drug powder. Then the powder is transferred for further pharmaceutical development, which often mill the powder to a particular particle size and granule to process into a tablet. Then the drug and polymer solution can be directly electrospun into a final product, thus creating a seamless process from chemical synthesis through creation of an appropriate final pharmaceutical product. This would eliminate powder handling of the drug substance, and possibly reduce variations in the final particle size of the drug substance during manufacturing. For colon targeting drug delivery approach, Eudragit ${ }^{\circledR}$ L 100-55 nanofibers loaded with diclofenac sodium were successfully prepared using an electrospinning process [22]. Green tea poly phenols (GTP) in Poly( $\varepsilon$-caprolactone) multi-walled carbon nanotubes composite nanofibers possessed a significant inhibition effect to tumor cells.GTP, as a multifunctional drug, encapsulated into polymer composite nanofibers, have broad application prospects in cancer therapy [23].

Nanomicelles: Nanomicelles, the self assemblies of block copolymers, are promising nanomedicaments for drug targeting and imaging as diagnostics. Stimulusresponsive site specific targeted nanomicelles are designed to release drugs based on stimuli like temperature, $\mathrm{pH}$, redox potential, ultrasound and magnetic field. Anticancer drug loaded nanomicelles are used to fulfill the challenges associated with cancer cells (e.g. Multidrug resistance, toxicity) for effective cancer treatment[24].Biocompatible, biodegradable and sterically stabilized phospholipid nanomicelles can improve cryopreservation of oral keratinocytes by promoting cell viability and plasma membrane integrity and act as a potential nanomedicine for cancer by selfassociated indisulam $[25,26]$.

Nanoshells: Nanoshells, another recent innovation in nanotechnology based pharmaceutical research, are miniscule beads coated with gold. By controlling the thickness of the layers making up the nanoshells, the beads can be designed to absorb specific wavelength of light. The most useful nanoshells are those which absorb the light nearer to infrared that can easily penetrate several centimetres in human tissues. This absorption of light by nanoshells creates an intense heat that is lethal to cells. Nanoshells can be employed in identification of cancer cells with association of antibodies. Lim et al., observed the heat generated by the light absorbing nanoshells, has successfully killed tumour cells while leaving neighboring cell intact [27]. Xie et al. studied in vivo PET imaging and biodistribution of radiolabeled gold nanoshells in rats with tumor xenograft which can be further applied to guide photo thermal treatment of cancer [28]. Another research explained the controlled drug delivery by temperature sensitive hydrogel with $\mathrm{SiO}_{2}-\mathrm{Au}$ nanoshells and layer-by-layer self assembled nanoshells $[29,30]$.

Magnetic nanopharmaceuticals: Magnetic Nanopharmaceuticals are the novel nanoparticulate pharmaceuticals that involve binding of the therapeutic or diagnostic part with magnetic nanoparticles (MNPs), such as oxidized iron or magnetite. The prerequisite for magnetic carriers used for biomedical applications is that, they must be aqua based, biocompatible, nontoxic and nonimmunogenic. In the versatility of various magnetic carriers used, iron oxide is the most commonly used carrier because of its biodegradability, biocompatibility, super paramagnetic effects and ability to serve as a contrast magnetic resonance imaging (MRI). Study demonstrates about the multifunctional magnetic nanoparticles employed for targeted drug 
delivery. It explains that a simple external magnetic field is all that is necessary to target a drug to a specific site inside the body without the need to functionalize the nanoparticles. However, the option to use magnetic targeting with external magnets on functionalized nanoparticles could prove as a more efficient means of drug delivery [31].

Metallic nanoparticles: The silver $(\mathrm{Ag})$, gold $(\mathrm{Au})$, copper $(\mathrm{Cu})$ and inorganic carriers such as silica, alumina that have been utilized for the preparation of nanoparticles, serve as a novel carriers for pharmaceutical formulations. Gold nanoparticles are most promising for drug delivery due to their excellent unique optical and photoelectric properties, inertness, nontoxicity, higher stability, ease of preparation, and possibility of bioconjugation and biomodification with various functional groups like thiols, disulfides and amines. These are applied in optical probes, targeted drug delivery and programmed material synthesis. The synergistic effects of antibacterial activity of silver nanoparticles with amoxicillin are studied along with wound dressing effect.

Nanosized powder: This nanosized powder is also called solid solution. Nanosized powders of gadoliniadoped ceria were prepared by the pyrolysis of a metal complex precursor using citric acid as complexant agent. The temperature of the gel decomposition should be suitably chosen to break up the porous structure formed, enabling the evolution of entrapped gaseous species. Sintered specimens with high densification may be obtained using this low-cost and relatively simple technique of synthesis [32].

Nanoemulsions: Nanoemulsions are basically class of multiphase colloidal dispersions system. Some lyotropic liquid crystalline phases, which are also known as 'micellar phases', 'mesophases', and 'microemulsions', have composition similar to nanoemulsions and nanoscale structure, but these phases are actually quite different in nature. Lyotropic liquid crystals are equilibrium structures comprised of liquids and surfactants, such as lamellar sheets, hexagonally packed columns, and micellar phases which form spontaneously through thermodynamic self assembly. In contrast, nanoemulsions do not form spontaneously; an external shear must be applied to rupture larger droplets into smaller ones. This is primarily because extreme shear, well beyond the reach of ordinary mixing devices, is applied to overcome the effects of surface tension to rupture the droplets into the nanoscale regime. Compared to micro emulsion phases, relatively little information is known about mechanism of creating and controlling nanoemulsions.
In the world of nanomaterials, nanoemulsions hold great promise as useful dispersions of deformable nanoscale droplets with optical properties ranging from opaque to nearly transparent. Moreover, it is very likely that nanoemulsions will play an increasingly important role commercially, since they can typically be formulated using significantly less surfactant than is required for nanostructured lyotropic microemulsion phases. A novel ultrasound-mediated chemotherapeutic modality is based on systemic injections of drug-loaded nanoemulsions that convert into microbubbles in situ under the action of therapeutic ultrasound. This modality proved very effective in inducing dramatic tumor regression in ovarian and pancreatic cancer models. Rapoport et al., reported the results of nanotherapy of ovarian, breast, and pancreatic cancerous tumors by paclitaxel-loaded nanoemulsions that convert into microbubbles locally in tumor tissue under the action of tumor-directed therapeutic ultrasound. Tumor accumulation of nanoemulsions was confirmed by ultrasound imaging. Dramatic regression of ovarian, breast, and orthotopic pancreatic tumors was observed in tumor therapy through systemic injections of drug-loaded nanoemulsions combined with therapeutic ultrasound, signifying efficient ultrasound-triggered drug release from tumoraccumulated nanodroplets. The drug release mechanism in the process of droplet-to-bubble conversion under the action of therapeutic ultrasound, nanodroplets converted into microbubbles from drugloaded nanoemulsions is studied [33]. A novel candesartan cilexetil loaded nanoemulsion was designed to improve the oral absorption of candesartan. The absorption of candesartan cilexetil loaded nanoemulsion was significantly improved in the intestinal tract compared with free drug. Candesartan cilexetil loaded nanoemulsion could be internalized into the enterocytes by clathrin-mediated endocytosis pathway, and then transported into the systemic circulation by portal vein and lymphatic pathway. The overall results indicated that nanoemulsion was effective for enhancing the oral absorption of candesartan, and its nanoemulsion demonstrated great potential for clinical application [34].

Nanosuspension: Nanosuspension is a biphasic system consisting of pure drug particles dispersed in an aqueous vehicle in which the diameter of the suspended particle is less than $1 \mu \mathrm{m}$ in size and stabilized by surfactants. Nanosuspensions can be used to enhance the solubility of drugs that are poorly soluble in aqueous as well as lipid media. As a result, the rate of flooding of the active compound increases and the maximum plasma level is reached faster. Nanosuspension have many advantages over conventional suspensions, such as increase in the 
dissolution velocity, saturation solubility of the drug, improved biological performance, long-term physical stability with ease of manufacture and scale-up. Dandagi et al., had made a comparative studies of controlled release of acyclovir novel ophthalmic nanosuspension, with traditional colloidal carriers of controlled release of acyclovir. A quassi-emulsion solvent evaporation method was adopted to prepare acyclovir loaded Eudragit RS 100 ophthalmic nanosuspension with the aim of improved ocular bioavailability and distribution. The study revealed that, nanosuspension has overcome the major disadvantages associated with colloidal carriers systems such as instability in cul de sac and short half life by increasing efficiency of drug encapsulation as well as by controlled release. Overall, the study proved that ophthalmic nanosuspension was capable of releasing the drug for a prolonged period of time and increased bioavailability [35].

\section{Toxicity Issues with Nanopharmaceuticals}

Along with endless opportunities in the field of pharmaceutical formulation, drug delivery, diagnostic and treatment of various diseases, the safety and toxicity parameters of nano based dosage formulations has biggest challenge for scientific community and for their commercialization. Various semi conductive metals like colloidal gold and iron oxide crystals which have been formulated in nano form have also demonstrated toxicity [36]. These particles easily penetrate the skin and blood brain barrier, as nanometric size range, causing severe toxicities. Apart from this, enhanced pulmonary toxicity has also been reported with these nano dosage formulations and therefore, more extensive research should be carried out to fully investigate any toxicity issues associated with these nanoformulations which draw the concern of pharmaceutical scientists all over the globe [37].

\section{Future Scope of Nanopharmaceutical Market}

It was predicated over a decade back that "nanopharmaceuticals will help prolonged life, improve its quality, and extend human physical capabilities" and by year 2015, half of all pharmaceutical productions, over $\$ 180$ billion per year would be based on nanotechnology. At present, nanoscale formulations and design of pharmaceutical dosage forms have become prerequisite to sustain the growth of the pharmaceutical industry. The market for nanopharmaceutical has made a rapid stair from $\$ 406$ million in 2004 to $\$ 3$ billion in 2009 and $\$ 16.6$ billion in 2015. According to a new report published by visiongain forecasts the world market for nanopharmaceuticals will reach $\$ 130$ billion in 2017.There is an active research and increasing demand of nanopharmaceutical applications resulting in better positions in the pharmaceutical and other health care industries [38, 39].

\section{Conclusion}

The review of literature and research of nanopharmaceuticals reveal that nanoparticulate systems have great potentials, being able to convert poorly soluble, poorly absorbed and labile biologically active substance into promising deliverable drugs. However, to optimize this nanopharmaceuticals delivery system, greater understanding of the different mechanisms of biological interactions, and particle engineering and characterization, is still essential. Further advances are needed in order to turn the concept of nanoparticle technology into a realistic practical application as the next generation of drug delivery system. In future, nanopharmaceuticals will greatly influence medical practice and healthcare because of their ability, in many cases, to shorten the time-market for active agents, extend the economic life of proprietary drugs and create additional revenue streams. The challenge of nanotechnology is to develop nanoparticles for biomedical and biotechnology applications, to deliver the pharmaceutical in the right place at the right time. Some formidable challenges include legal, environmental, safety, ethical and regulatory questions as well as emerging thickets of overlapping patent claims. Though there are few nanopharmaceuticals in the market that have been approved by the FDA, these formulations have immense impact in the field of therapy management and also hold greater promise in pharmaceutical drug design and delivery.

\section{References}

1. Gaur A, Bhatia AL (2008) Nanopharmaceuticals: An overview. Asian J Exp Sci 22(2): 51-62 .

2. Gupta BR, Kompella BU (2006) Fundamentals of Drug Nanoparticles: In Nanoparticle Technology for Drug Delivery. New York: Taylor \& Francis 1-18.

3. Bawa R (2008) Nanoparticle based therapeutics in Human-A Survey. Nanotechnology laws \& business 5(2): 135-155.

4. Saha M (2009) Nanomedicine: Promising Tiny machine for the healthcare for the future-A Review. Oman Med J 24(4): 242-247.

5. Bawa R (2009) Nanopharmaceuticals for Drug delivery-A review. Touch Briefings 6: 122-127. 
6. Vladimir PT (2006) Nanoparticulate as Drug Delivery. Imperial College Press 499-506.

7. Basu B, Garala K, Bhalodi R, Joshi B, Mehta K (2010) Solid lipid nanoparticles: A promising tool for drug delivery system. J Pharm Res 3(1): 84-92.

8. Blasi P, Giovagnoli S, Schoubben A, Ricci M, Rossi C (2007) Solid lipid nanoparticles for targeted brain drug delivery. Adv Drug Deliv Rev59 (6): 454-477.

9. Subedi RK, Kang KW, Choi H (2009) Preparation and characterization of solid lipid nanoparticles loaded with doxorubicin. Eur J Pharm Sci 37(3-4): 508-513.

10. Moritz B, Ruppert C, Schmeh T, Guenther A, Betz T et al. (2011) Biophysical investigation of pulmonary surfactant surface properties upon contact with polymeric nanoparticles in vitro. Nanomedicine $7(3): 341-350$.

11. Dutta P, Dey J (2011) Drug solubilization by amino acid based polymeric nanoparticles: Characterization and biocompatibility studies. Int J Pharm 421: 353-363.

12. Donald EO, Peppas NA (2006) Opsonization, biodistribution, and pharmacokinetics of polymeric nanoparticles. Int J Pharm 307(1): 93-102.

13. Zhang X, Gan Y, Gan L, Nie S, Pan W (2008) PEGlyated nanostructured lipid carriers loaded with 10-hydroxycamptothecin: An efficient carrier with enhanced anti-tumour effects against lung cancer. J Pharm Pharmacol 60(8): 1077-1087.

14. Kayser 0, Lemke A, Hernandez-Trejo (2005) The impact of nanobiotechnology on the development of new drug delivery systems. Curr Pharm Biotechnol 6(1): $3-53$.

15. Shegokar R, Rainer HM (2010) Nanocrystals: Industrially feasible multifunctional formulation technology for poorly soluble actives. Int J Pharm 399(1-2): 129-139.

16. Deng J, Huang L, Liu F (2010) Understanding the structure and stability of paclitaxel nanocrystals. Int J Phar 390(2): 242-249.

17. Piao H, Ouyang M, Xia D, Quan P, Xiao W, et al. (2011) In vitro-in vivo study of CoQ10-loaded lipid nanoparticles in comparison with nanocrystals. Int J Pharm 419: 255-259.
18. Yang W, Cheng Y, Xu T, Wang WL, Wen LP (2009) Targeting cancer cells with biotin dendrimer conjugates. Eur J Med Chem 44(2): 862-868.

19. Li M, Choi SK, Thomas TP, Desai A, Lee K, et al. (2011) Dendrimer-based multivalent methotrexates as dual acting nanoconjugates for cancer cell targeting. Eur J Med Chem 1-13.

20. Firme III CP, Bandaru PR (2010) Toxicity issues in the application of carbon nanotubes to biological systems. Nanomedicine 6(2): 245-256.

21. Foldvari M, Bagonluri M (2008) Carbon nanotubes as functional excipients for nanomedicines: $\mathrm{I}$. pharmaceutical properties. Nanomedicine 4(3): 173-182.

22. Shen $X$, Yu D, Zhu L, Branford-White C, White K, et al. (2011) Electrospun diclofenac sodium loaded Eudragit ${ }^{\circledR}$ L 100-55 nanofibers for colon-targeted drug delivery. Int J Pharm 408(1-2): 200-207.

23. Shao S, Li L, Yang G, Li J, Luo GT, et al. (2011) Controlled green tea polyphenols release from electrospun PCL/MWCNTs composite nanofibers. Int J Pharm 421(2): 310-320.

24. Zhang W , Huang J , Fan N , Yu J , Liu Y, et al. (2010) Nanomicelle with long term circulation and enhanced stability of camptothecin based on mPEGlated $\alpha$, $\beta$-poly(L-aspartic acid) campothecin conjugates. Colloid Surface B 81(1): 297-303.

25. Rubinstein I, Onyüksel H (2007) Biocompatible, biodegradable and sterically stabilized phospholipid nanomicelles improve cryopreservation of oral keratinocytes: A preliminary investigation. Int J Pharm 338: 333335.

26. Cesur H, Rubinstein I, Pai A, Onyuksel H (2009) Selfassociated indisulam in phospholipid-based nanomicelles: A potential nanomedicine for cancer. Nanomedicine 5(2): 178-183.

27. Lim Y, Park O, Jung HT (2003) Gold nanolayer encapsulated silica nanoparticles synthesized by surface seeding and shell growing method:near infrared responsive materials. J Colloid Interface Sci 263(2): 449-453.

28. Xie H, Wang ZJ, Bao A, Goins B, Phillips WT (2010) In vivo PET imaging and biodistribution of radiolabeled gold nanoshells in rats with tumor xenografts . Int J Pharm 395(1-2): 324-330. 
29. Bikram M, Gobin AM, Whitmire RE, West JL (2007) Temperature-sensitive hydrogels with $\mathrm{SiO}_{2}-\mathrm{Au}$ nanoshells for controlled drug delivery. J Controlled Release 123(3): 219-227.

30. Katsuhiko A, Yuri ML, Kawakami K Ji Q, Hill JP (2011) Layer-by-layer self-assembled shells for drug delivery. Adv Drug Deliv Rev 63: 762-771.

31. Kumar A, Jena PK, Behera S, Lockey RF, Mohapatra $S$, et al. (2010) Multifunctional magnetic nanoparticles for targeted delivery. Nanomedicine 6(1): 64-69.

32. Rocha RA, Muccilo ENS (2003) Physical and chemical properties of nanosized powders of gadolinia-doped ceria prepared by the cation complexation technique. Mater Res Bull 38: 19791986.

33. Rapoport NY, Kennedy AM, Shea JE, Scaife CL, Nam $\mathrm{KH}$ (2009) Controlled and targeted tumor chemotherapy by ultrasound-activated nanoemulsions/microbubbles. J Controlled Release 138(3): 268-276.

34. Gao F, Zhang Z, Bu H, Huang Y, Gao Z (2011) Nanoemulsion improves the oral absorption of candesartan cilexetil in rats: Performance and mechanism. J Controlled Release 149(2): 168-174.

35. Dandagi P, Keru S, Mastiholimath V, Gadad A, Kulkarni A (2009) Polymeric ocular nanosuspension for controlled release of acyclovir: in vitro release and ocular distribution. Iran J Pharm Res 8: 79-86.

36. A Shvedova, A Pietroiusti, V Kagan (2016) Nanotoxicology ten years later: lights and shadows. Toxicol Appl Pharmacol 299: 1-2.

37. Soto K, Carrasco A, Powell TG, Garza KM, Murr LE (2005) Comparative in vitro cytotoxicity assessment of some manufactured nanoparticulate materials characterized by transmission electron microscopy. J Nanoparticle Res 7: 145-169.

38. Bawa R, Srikumaran Melethil, William J Simmons, and Drew Harris (2008) Nanopharmaceuticals: Patenting issues and FDA regulatory challenges. The SciTech Lawyer 5(2): 1-6.

39. Gadad A, Soni A, Dandagi P, Mastiholimath V (2011) Nanotechnology in drug delivery: A review. Indian Drug 48: 5-15. 